\title{
A Fruit Sensing and Classification System by Fractional Fourier Entropy and Improved Hybrid Genetic Algorithm
}

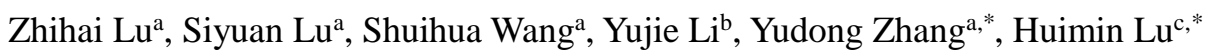 \\ ${ }^{a}$ School of Computer Science and Technology, Nanjing Normal University, Nanjing, Jiangsu 210023, China \\ ${ }^{\mathrm{b}}$ School of Information Engineering, Yangzhou University, Yangzhou, Jiangsu 225009, China \\ ${ }^{c}$ Department of Mechanical and Control Engineering, Kyushu Institute of Technology, Fukuoka Prefecture 8048550, \\ Japan
}

*Corresponding Author: Y. Zhang (zhangyudong@ njnu.edu.cn) and H Lu (dr.huimin.lu@ieee.org)

\begin{abstract}
It remains a challenge to classify different categories of fruits because of the similarities of shape, color, and texture among them. We presented a novel approach in order to classify fruits accurately and efficiently based on computer vision techniques. We obtained the coefficients using fractional Fourier transform. The entropies extracted from the coefficients were fed into the classifier as the features. A multilayer perceptron optimized by an improved hybrid genetic algorithm was used as the classifier. The experiment results on 1653 fruit images demonstrated that the proposed method achieved an overall accuracy of $89.59 \%$, which was superior to the state-of-art approaches. Our method is efficientive in identifying fruit categories.
\end{abstract}

Keywords: fruit classification; fractional Fourier entropy; multilayer perceptron; genetic algorithm; machine learning.

\section{Introduction}

Automatic fruit classification can help in factory production, supermarket selling, fruit-picking robot, etc. Nevertheless, there is no practical method for automatic fruit classification.

Pennington (2009) [1] employed clustering algorithm to classify fruits and vegetables. Pholpho (2011) [2] utilized visible spectroscopy to classify non-bruised and bruised longan fruits. The classification models combined the principal component analysis (PCA), partial least square discriminant analysis and soft independent modeling of class analogy. Yang (2012) [3] applied multispectral imaging analysis to the blueberry yield estimation system.
Wu (2012) [4] suggested the max-wins-voting SVM with Gaussian RBF kernel to classify different categories of fruits, with overall accuracy of $88.2 \%$. Feng (2013) [5] utilized Raman spectroscopy which was a rapid and non-destructive tool, and chose a polynomial fitting for baseline correction. Afterwards, PCA and hierarchical cluster analysis (HCA) were employed to classify eight different citrus fruits. Cano Marchal (2013) [6] established an expert system on the strength of computer vision to estimate the content of impurities in olive oil samples. Breijo (2013) [7] used an odor sampling system (electronic nose) for classification of the aroma of Diospyros kaki. Fan (2013) [8] used an artificial neural network to predict the texture characteristics from extrusion food surface images. Omid (2013) [9] developed an intelligent system based on combined fuzzy logic and machine vision techniques for grading of egg using parameters as defects and size of eggs. $\mathrm{Ji}$ (2014) [10] used a fitness-scaling chaotic ABC (FSCABC) algorithm to develop an automatic fruit classification system, which can identify 18 kinds of fruits. Ji (2015) [11] presented a novel fruit classification system based on wavelet entropy.

The aim of this study was to present a new approach for fruit classification. We extracted the fractional Fourier entropy (FRFE) from the fruit images to form the feature vector, which greatly reduced the feature space. An improved hybrid genetic algorithm was employed to optimize the multilayer perceptron (MLP), which was used as the classifier. With this develop method, we achieved better results than existing methods.

The structure of the remainder is organized as follows: Section 2 is about the materials used in the experiment. 
Section 3 describes the fractional Fourier entropy. Section 4 presents the multilayer perceptron classifier. Section 5 shows the improved genetic algorithm, which is used to obtain the optimal parameters of the MLP. Section 6 discusses the results and the contribution of this study. Final Section 7 is devoted to conclusions.

\section{Materials}

The "fruit" dataset was acquired by both 6 months of on-site collecting via digital camera (See Figure 1) and search engine (Google). The 18 types of fruits and their numbers can be found in literature [12].

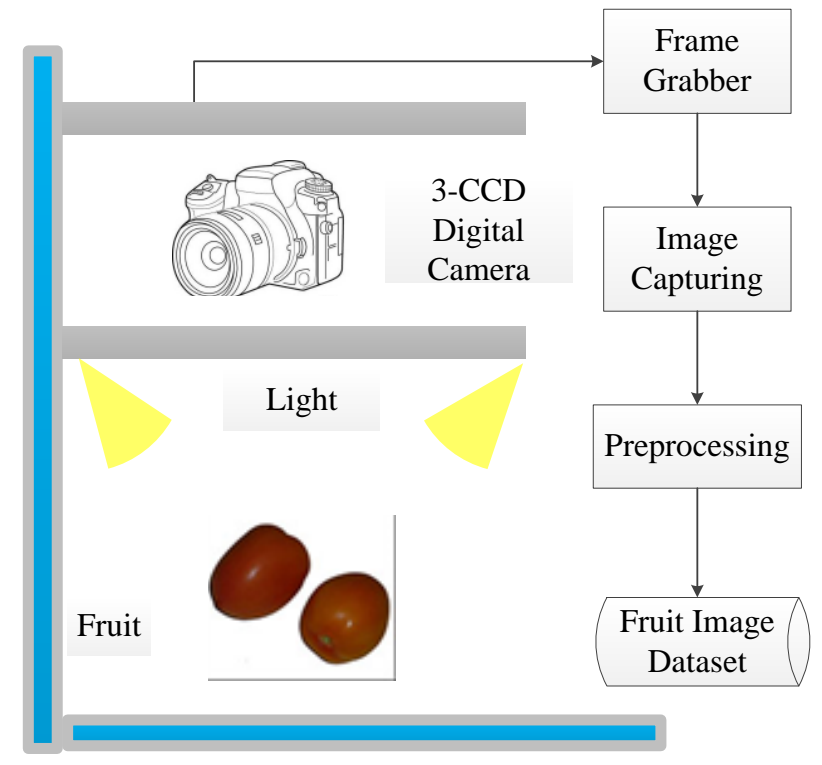

Fig. 1 Fruit image acquiring system

\section{Fractional Fourier Entropy}

The fractional Fourier entropy (FRFE) is one of the global features of an image, proposed by Cattani (2016) [13]. It is obtained by performing 2-D fractional Fourier transform (FRFT) over an image and extracting the Shannon entropy of the coefficient [14-16].

For a given function $y(t)$, the FRFT with $\alpha$-angle is defined as $\gamma_{\alpha}$ with the equation below:

$$
\gamma_{\alpha}(v)=\int_{-\infty}^{+\infty} P_{\alpha}(t, v) y(t) d t
$$

Where $v$ represents the frequency, and $t$ denotes time. $P$ is the transform kernel function [17]:

$$
\begin{aligned}
& P_{\alpha}(t, v)=\sqrt{1-i \cot \alpha} \times \\
& \exp \left(i \pi\left(v^{2} \cot \alpha-2 v t \csc \alpha+t^{2} \cot \alpha\right)\right)
\end{aligned}
$$

Where $i$ denotes the imaginary unit. 2D-FRFT can be easily implemented by 1D-FRFT . Two angles, $\alpha$ and $\beta$ are needed for 2D-FRFT, denoted by $\gamma_{\alpha, \beta}$.

Next, we calculate entropy over the 2D-FRFT decomposition result. Suppose $R$ is discrete and random, and it falls within the value set $\left(r_{1}, r_{2}, \ldots, r_{n}\right)$ with probability mass function of $G(R)$, we have

$$
Z(R)=F[I(R)]=F[-\ln (G(R))]
$$

Where $F$ represents expected value and $Z$ is the entropy. Generally, we have

$$
Z(R)=-\sum_{i=1}^{n} G\left(r_{i}\right) \log G\left(r_{i}\right)
$$

Therefore, the FRFE of a fruit image, denoted by $\mathrm{E}$ is defined as:

$$
E(I)=Z\left[\gamma_{\alpha, \beta}(I)\right]
$$

\section{Classifier-Multilayer Perceptron}

Multilayer perceptron (MLP) is a kind of feedforward neural network with one input layer, one output layer, and several hidden layers [18]. As the structure of MLP is flexible, we need to define the architecture of the MLP before training it for optimal weights/biases. [19] MLP can be used for classification and function approximation, and the performance can be superior to support vector machine, if the architecture is defined properly.

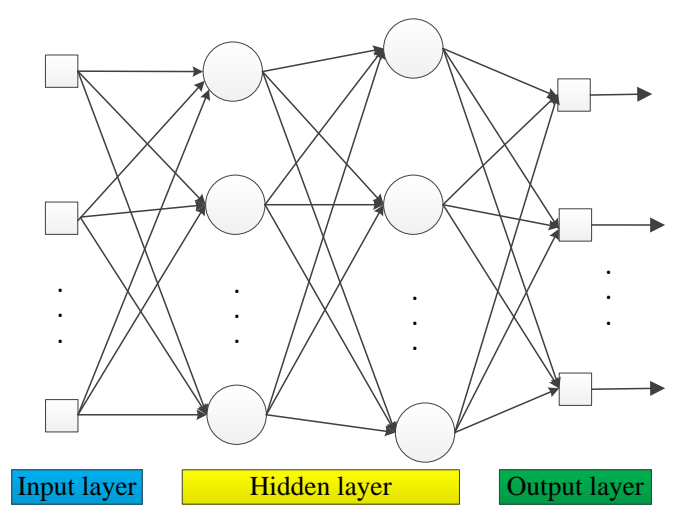

Fig. 1 Architecture of an MLP

Fig. 1 shows the structure of an MLP, it consists of one input layer, one hidden layers, and one output layer. The structure of an MLP is dependent on the given problem [20]. Generally, the number of input neurons equals to the dimension of the feature vector, and the number of output nodes is set as the number of class [21]. The weights/biases 
of the MLP are obtained by training [22]. Many optimization algorithms have been applied, such as back propagation (BP), and particle swarm optimization (PSO) [23].

However, these methods are prone to get trapped in local extrema. Hence, it's still difficult to achieve the optimal solution. Moreover, determining the number of hidden neuron is another challenge, So far, there is no general answer to the problem of defining the structure of hidden layer.

\section{Improved Genetic Algorithm}

We employed improved hybrid genetic algorithm to train the MLP to obtain the optimal weights/biases.

\subsection{Standard Genetic Algorithm}

Standard genetic algorithm (GA) is widely used for optimization. GA follows the criteria that survival of the fittest, which mimics the process of natural selection [24]. It searches through a population of candidate solutions to the problem, called individuals, by iteration. Each candidate solution has its own chromosomes, which are randomly initialized, and can be mutated and altered during the iterations [25].

The population of the individuals in each iteration is denoted as generations. The value of the objective function of each individual is regarded as the fitness of it [26-28]. During each generation, the fitness of each individual is calculated. The more fit individuals are selected to breed the next generation, while the remaining individuals are eliminated [29]. The new generation are formed by modifying crossover and randomly mutation of the genome of the more fit individuals. The algorithm will terminate if a candidate solution that satisfies minimum criteria is found or it reached the fixed number of generations [30].

However, GA has a poor ability of local search, which means that it is unable to adjust well the candidate solution near the potential region. Meanwhile, it's also often-observed that the quality of the offspring worsens with the generations.

\subsection{Improved hybrid genetic algorithm}

Improved hybrid genetic algorithm is proposed by Ahmad (2013) [31] to optimize the number of hidden nodes, weights, and feature subset in MLP [32]. Firstly, it encodes the candidate solution into the chromosome, containing 3 gene segments in binary format, as is shown in Fig. 2.

\begin{tabular}{|c|c|c|}
\hline GS1 & GS2 & GS3 \\
\hline $0100 \ldots$ & $1100 \ldots$ & $1010 \ldots$ \\
\hline $\begin{array}{c}\text { Random initial } \\
\text { weight generator }\end{array}$ & $\begin{array}{c}\text { No of hidden } \\
\text { nodes }\end{array}$ & Feature subset \\
\hline
\end{tabular}

Fig. 2. The chromosome that represents the MLP

The value of fitness $F$ of MLP is defined as:

$$
F=r \times a+(1-r) \times \frac{1}{c}
$$

where $r \in(0,1]$, is the weight assigned to weigh the contributions of the testing accuracy $(a)$ and the inverse complexity $(1 / c)$ of MLP, given as:

$$
\begin{gathered}
a=\left(\frac{n_{c}}{n_{t}}\right) \times 100 \\
c=(o \times p)+(p \times q)+p+q
\end{gathered}
$$

Here, $n_{t}$ denotes the total number of the testing samples, $n_{c}$ the number of samples correctly classified, $o$ the number of chosen feature, $p$ the number of hidden nodes, $q$ the number of output neurons, respectively.

Segmented multi-chromosome crossover (SMCC) is used, which can produce a chromosome that contains gene from more than one couple chromosome. The basic steps of the improved hybrid genetic algorithm are illustrated in Table 1. The whole diagram of IHGA is shown in Fig. 3.

Table 1 Pseudocode of the improved hybrid genetic algorithm

Improved Hybrid Genetic Algorithm

Step 1: Randomly initialize the population chromosomes.

Step 2: Decode each chromosome.

Step 3: Build the MLP with the parameters obtained, and start training.

Step 4: Calculate the fitness of the MLP.

Step 5: Create the elite chromosome and SMCC chromosomes.

Step 6: Directly copy the best individual obtained to the next generation.

Step 7: Crossover and mutate the members of elite and normal pool to form the next generation.

Step 8: Repeat Step 2 to 7 until the maximum generation, and then output the optimal solution. 


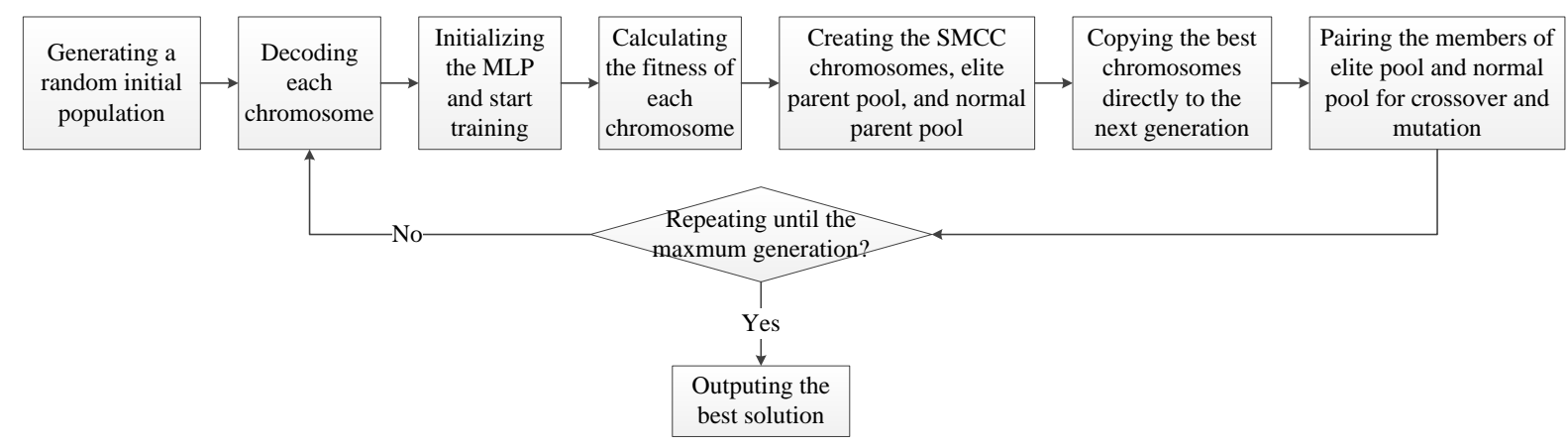

Fig. 3 Diagram of IGHA

\section{Experiments and Results}

\subsection{FRFE Result}

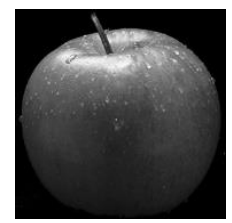

Fig. 4 An apple image

We take an apple image as an example as shown in Fig. 4. We take 25 difference combinations of $(\alpha, \beta)$. The results are shown in Fig. 5. The results over other types of fruits are not displayed, due to limited page.

Those 25 FRFEs were submitted to the proposed classifier - IGHA-MLP. The IGHA iterates to update the hidden neuron number, classifier weights, and selected features at every step. The parameters were set by experience as listed in Table 2.

Table 2 Parameter Setting

Parameter Value

0.6

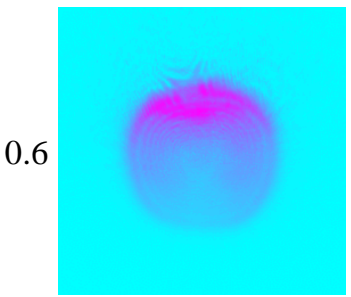

0.7

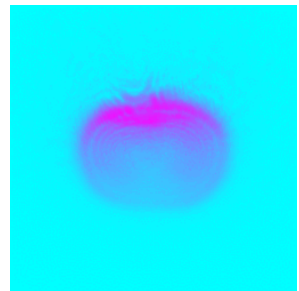

0.8

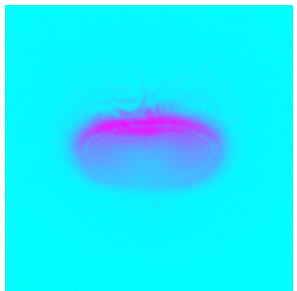

\begin{tabular}{ll}
\hline Population & 20 \\
Max-generation & 40 \\
Elite chromosome & 5 \\
SMCC chromosome & 15 \\
Elite parent pool & 9 \\
Normal parent pool & 9 \\
Elitism & 2 \\
Mutation probability & 0.1 \\
Crossover probability & 0.6 \\
$R$ & 0.8 \\
\hline
\end{tabular}

Population represents the size of individuals, Max-generation stands for the maximum iteration generations. In every generation, the best 5 chromosome are selected as the Elite chromosome, while the rest 15 are used as SMCC chromosome according to the fitness value. The elite and normal parent pools both contain 9 chromosomes. We set the elitism as 2 , which means 2 individuals are copied to the next generation directly. The mutation rate and crossover rate are 0.1 and 0.6 , respectively. The $r$ is the parameter in Equation (6), used to weigh the contributions of the testing accuracy and the inverse complexity of MLP. 


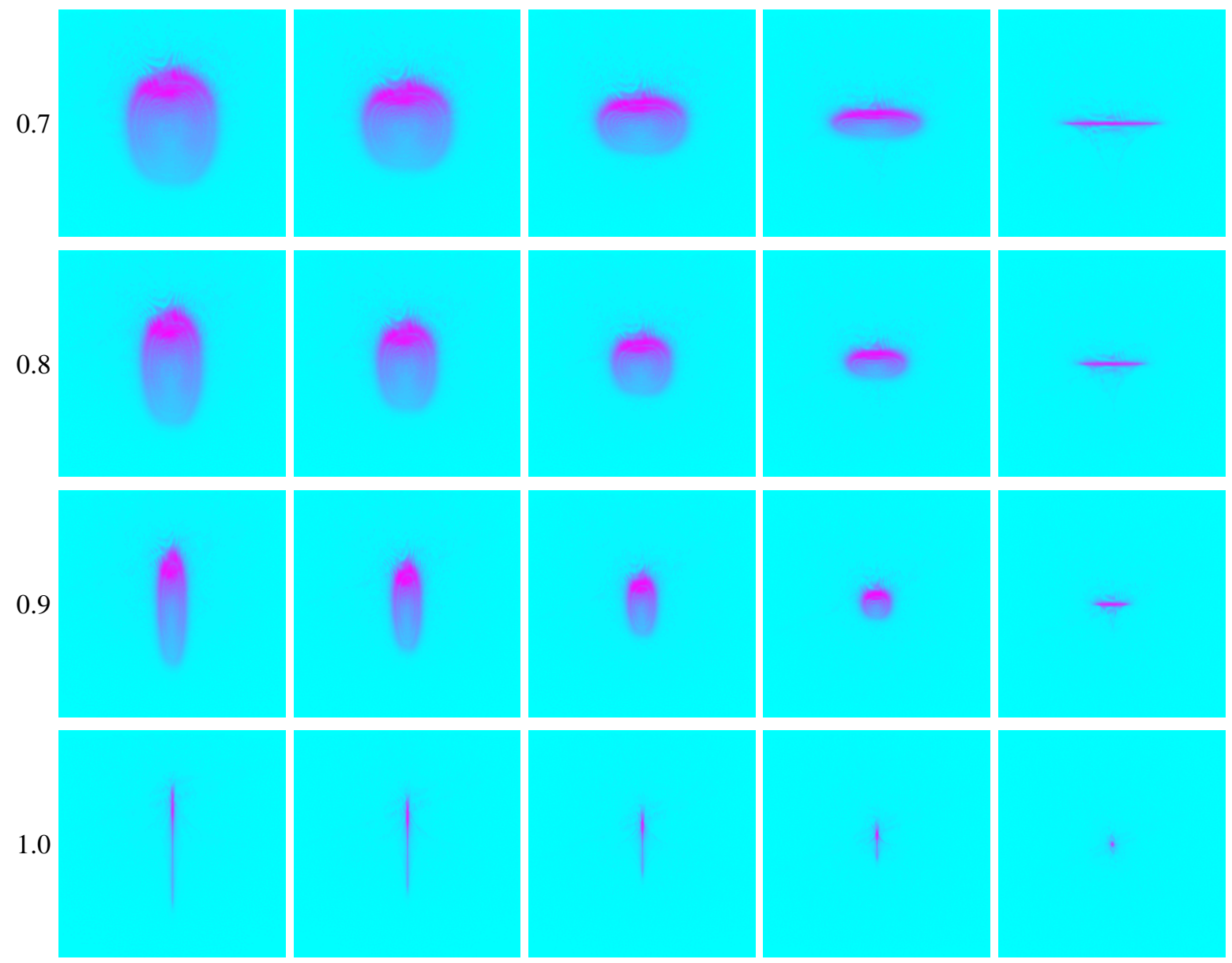

Fig. 5 FRFE Result

\subsection{Comparison between GA and IHGA}

In the third experiment, we employed FRFE to extract features, and then used the GA to train MLP and compare the results with IHGA. The results are listed in Table 3. Here the GA only obtains an accuracy of $87.42 \%$, and IHGA obtains an accuracy of $89.59 \%$.

Table 3 Comparison between GA and IHGA

\begin{tabular}{ll}
\hline Training Method & Accuracy \\
\hline GA & $87.42 \%$ \\
IHGA (Proposed) & $\mathbf{8 9 . 5 9 \%}$ \\
\hline
\end{tabular}

IHGA is better than GA. The reason lies in the chromosome that consists of three segments: weights, hidden neuron number, and feature subset. Meanwhile, the fitness takes both testing accuracy and the complexity of the MLP structure into consideration. Moreover, SMCC used in the experiment enables that the produced chromosome contains gene from more than one couple chromosome, which benefits the evolution process.

\subsection{Classification Performance}

The final classification performance was listed in Table 4, and comparison with state-of-the-art approaches was also showed. We see that the $(\mathrm{CH}+\mathrm{MP}+\mathrm{US})+\mathrm{PCA}$ + kSVM [4] used 14 features and obtained an accuracy of $88.20 \%,(\mathrm{CH}+\mathrm{MP}+\mathrm{US})+\mathrm{PCA}+$ FSCABC-FNN [10] used 14 features and obtained an accuracy of $89.11 \%$. WE + PCA + FSCABC-FNN [11] used 12 features and obtained an accuracy of $89.47 \%$, WE + PCA + BBO-FNN [11] used 12 features and obtained an accuracy of $89.47 \%,(\mathrm{CH}+\mathrm{MP}$ + US) + PCA + BBO-FNN [12] used 14 features and obtained an accuracy of $89.11 \%$. Finally, this proposed FRFE + IHGA-MLP method used 25 features and yielded the largest accuracy of $89.59 \%$.

Table 4 Classification performance

\begin{tabular}{lll}
\hline Approach & $\begin{array}{l}\text { Feature } \\
\text { Number }\end{array}$ & Accuracy \\
\hline$(\mathrm{CH}+\mathrm{MP}+\mathrm{US})+\mathrm{PCA}+$ & 14 & $88.20 \%$ \\
\hline
\end{tabular}




\begin{tabular}{lll}
\hline kSVM [4] & \\
$(\mathrm{CH}+\mathrm{MP}+\mathrm{US})+\mathrm{PCA}+14$ & $89.11 \%$ \\
FSCABC-FNN [10] & & \\
$\mathrm{WE}+\mathrm{PCA}+\mathrm{FSCABC-FNN}$ & 12 & $89.47 \%$ \\
{$[11]$} & & $89.47 \%$ \\
$\mathrm{WE}+\mathrm{PCA}+\mathrm{BBO}-\mathrm{FNN}[11]$ & 12 & $89.11 \%$ \\
$(\mathrm{CH}+\mathrm{MP}+\mathrm{US})+\mathrm{PCA}+14$ & $\mathbf{8 9 . 5 9 \%}$ \\
BBO-FNN [12] & & \\
FRFE + IHGA-MLP (Proposed) & 25 & \\
\hline
\end{tabular}

(Bold means the best)

Results in Table 4 suggested that the proposed method was the only one that didn't use PCA which was an effective feature reduction tool. Though 25 was the most feature number among the listed approaches, it was not too many for computers nowadays, and the time for PCA was saved.

Meanwhile, the proposed method achieved the highest accuracy of $89.59 \%$, and FSCABC-FNN and BBO-FNN came the second and third with the same accuracy of $89.47 \%$, which indicated the superiority of FRFT + IHGA-MLP to other approaches. The worst classifier was kSVM with $88.20 \%$ accuracy. That's because the performances of FNN and MLP exceeded KSVM when large datasets were available [33, 34].

The limitations of the proposed approach lie in that the weights/biases of MLP are not understandable for common food engineering experts. Besides, the classification performance can be better with improved fitness function.

\section{Conclusion}

In this study, we proposed a new approach for fruit classification, combining FRFE and MLP optimized by improved hybrid GA. The proposed method yielded an overall accuracy of $89.59 \%$ in identifying 1653 fruits of 18 different categories, which was higher than state-of-art methods.

\section{Acknowledgment}

This paper is supported by Leading Initiative for Excellent Young Researcher (LEADER) of Ministry of Education, Culture, Sports, Science and Technology-Japan (16809746), Natural Science Foundation of China (61602250), Natural Science Foundation of Jiangsu Province (BK20150983), Open Program of Jiangsu Key Laboratory of 3D Printing Equipment and Manufacturing
(3DL201602).

\section{References}

(1) Pennington, J.A.T. and R.A. Fisher, "Classification of fruits and vegetables". Journal of Food Composition and Analysis, 2009. 22(Supplement 1): pp. S23-S31

(2) 2Pholpho, T., et al., "Classification of longan fruit bruising using visible spectroscopy". Journal of Food Engineering, 2011. 104(1): pp. 169-172

(3) Yang, C., et al., "Classification of blueberry fruit and leaves based on spectral signatures". Biosystems Engineering, 2012. 113(4): pp. 351-362

(4) Wu, L. and Y. Zhang, "Classification of Fruits Using Computer Vision and a Multiclass Support Vector Machine". Sensors, 2012. 12(9): pp. 12489-12505

(5) Feng, X.W., et al., "Rapid Classification of Citrus Fruits Based on Raman Spectroscopy and Pattern Recognition Techniques". Food Science and Technology Research, 2013. 19(6): pp. 1077-1084

(6) Cano Marchal, P., et al., "Expert system based on computer vision to estimate the content of impurities in olive oil samples". Journal of Food Engineering, 2013. 119(2): pp. 220-228

(7) Breijo, E.G., et al., "Odour sampling system with modifiable parameters applied to fruit classification". Journal of Food Engineering, 2013. 116(2): pp. 277-285

(8) Fan, F.H., et al., "Prediction of texture characteristics from extrusion food surface images using a computer vision system and artificial neural networks". Journal of Food Engineering, 2013. 118(4): pp. 426-433

(9) Omid, M., et al., "An expert egg grading system based on machine vision and artificial intelligence techniques". Journal of Food Engineering, 2013. 118(1): pp. 70-77

(10) Ji, G., "Fruit classification using computer vision and feedforward neural network". Journal of Food Engineering, 2014. 143: pp. 167-177

(11) Ji, G., et al., "Fruit classification by wavelet-entropy and feedforward neural network trained by fitness-scaled chaotic $\mathrm{ABC}$ and biogeography-based optimization". Entropy, 2015. 17(8): pp. 5711-5728

(12) Wu, J., "Fruit classification by biogeography-based optimization and feedforward neural network". Expert Systems, 2016. 33(3): pp. 239-253

(13) Cattani, C. and R. Rao, "Tea Category Identification Using a Novel Fractional Fourier Entropy and Jaya 
Algorithm”. Entropy, 2016. 18(3), Article ID: 77

(14)Chen, S., et al., "Magnetic resonance brain image classification based on weighted-type fractional Fourier transform and nonparallel support vector machine". International Journal of Imaging Systems and Technology, 2015. 25(4): pp. 317-327

(15) Li, J., "Detection of Left-Sided and Right-Sided Hearing Loss via Fractional Fourier Transform". Entropy, 2016. 18(5), Article ID: 194

(16) Yang, X., et al., "Pathological Brain Detection by a Novel Image Feature-Fractional Fourier Entropy". Entropy, 2015. 17(12): pp. 8278-8296

(17) Liu, G., "Computer-aided diagnosis of abnormal breasts in mammogram images by weighted-type fractional Fourier transform". Advances in Mechanical Engineering, 2016. 8(2), Article ID: 11

(18) Sun, Y., "A Multilayer Perceptron Based Smart Pathological Brain Detection System by Fractional Fourier Entropy". Journal of Medical Systems, 2016. 40(7), Article ID: 173

(19) Ahmad, M.T., et al., "Fast multilayer perceptron neural network-based control algorithm for shunt compensator in distribution systems". IET Generation Transmission \& Distribution, 2016. 10(15): pp. 3824-3833

(20) Wang, S.-H. "Single slice based detection for Alzheimer's disease via wavelet entropy and multilayer perceptron trained by biogeography-based optimization". Multimedia Tools and Applications, 2016, DOI: 10.1007/s11042-016-4222-4 (Online).

(21) Ramchoun, H., et al., "Multilayer Perceptron: Architecture Optimization and Training". International Journal of Interactive Multimedia and Artificial Intelligence, 2016. 4(1): pp. 26-30

(22) Ebtehaj, I., et al., "A nonlinear simulation method based on a combination of multilayer perceptron and decision trees for predicting non-deposition sediment transport". Water Science And Technology-Water Supply, 2016. 16(5): pp. 1198-1206

(23) Ji, G., "A comprehensive survey on particle swarm optimization algorithm and its applications". Mathematical Problems in Engineering, 2015, Article ID: 931256

(24) Wei, L. and J. Yang, "Fitness-scaling adaptive genetic algorithm with local search for solving the Multiple Depot Vehicle Routing Problem". SIMULATION, 2016. 92(7): pp. 601-616

(25) Galan, C.O., et al., "Missing data imputation of questionnaires by means of genetic algorithms with different fitness functions". Journal of Computational And Applied Mathematics, 2017. 311: pp. 704-717

(26)Zhang, Y., et al., "Genetic Pattern Search and Its Application to Brain Image Classification". Mathematical Problems in Engineering, 2013, Article ID: 580876

(27) Shokrollahi, A. and B.M.N. Maybodi, "An Energy-Efficient Clustering Algorithm Using Fuzzy C-Means and Genetic Fuzzy System for Wireless Sensor Network". Journal of Circuits Systems and Computers, 2017. 26(1): p. 22, Article ID: 1750004

(28) Ji, G.L., "A Rule-Based Model for Bankruptcy Prediction Based on an Improved Genetic Ant Colony Algorithm". Mathematical Problems in Engineering, 2013, Article ID: 753251

(29) Tan, J.Z. and W.L. Kerr, "Determination of glass transitions in boiled candies by capacitance based thermal analysis (CTA) and genetic algorithm (GA)". Journal of Food Engineering, 2017. 193: pp. 68-75

(30) Barros, F.G.N., et al., "Compression of electrical power signals from waveform records using genetic algorithm and artificial neural network". Electric Power Systems Research, 2017. 142: pp. 207-214

(31) Ahmad, F., et al., "Intelligent medical disease diagnosis using improved hybrid genetic algorithm--multilayer perceptron network". J Med Syst, 2013. 37(2): p. 9934

(32) Lu, S., "A note on the weight of inverse complexity in improved hybrid genetic algorithm". Journal of Medical Systems, 2016. 40(6), Article ID: 150

(33) Li, Q., et al., "Predicting hourly cooling load in the building: A comparison of support vector machine and different artificial neural networks". Energy Conversion and Management, 2009. 50(1): pp. 90-96

(34) Pan, S., et al., 'Parkinson's Disease tremor classification - A comparison between Support Vector Machines and neural networks". Expert Systems with Applications, 2012. 39(12): pp. 10764-10771 